\title{
A Design of Lightweight Distributed GIS
}

\author{
Weng Yu ${ }^{1, \mathrm{a}}$,Cheng Wenyi ${ }^{2, \mathrm{~b}}$ \\ ${ }^{1}$ College of Information Engineering, Minzu University of China, Beijing, \\ 100081,China \\ ${ }^{2}$ College of Information Engineering, Minzu University of \\ China ,Beijing,100081, China \\ adr_wengyu@126.com,bflyingdoor@foxmail.com
}

\begin{abstract}
.
In order to display and observe some special data on a map,the researchers proposes a lightweight and effective Geographic Information System(GIS) over distributed environment, which can be integrated in other cloud computing system easily. The lightweight distributed geographic information system (LDGIS) acted as a service on the distributed platform. The researchers developed a Web Map Service (WMS) program conducting large tile file to service .The GIS provided essential functions such show maps and Data ,while further options like drawing and editing are not given. The researcher will show how to construct and implement this System in this paper.
\end{abstract}

Keywords-Distributed geographic Information System; GIS; Local WMS; Lightweight GIS.

\section{Introduction}

In the wake of the mushrooming of information and knowledge we human acquire,the data loaded on map increasing rapidly ,covers extensive areas. Nowadays ,GIS is widely used in agriculture,animal husbandry,scientific research,exploration,military,civil use[1]. How to organize and integrate vast data is a big challenge to researcher [2].

We designed a platform to integrate different fields of data concerned with ethnic minorities of China. In order to provide direct observation,wo proposed 
this lightweight distributed GIS. We want to exhibit a mass of data marked with geographic meta tags,but have no requirement in further map edite option nor multiplex correspondence protocol. As a service on a cloud platform ,our GIS must be independent enough to some extent and easy to deploy and remove.

We researched the general Web GIS and seek to find a feasible solution for our conceive. Existing GIS are mostly served on workstations, seldomly of these are primarily support distributed system. After studying the rationale of several famous GIS instance and tools,we proposed our design[3].

In part II we will introduce some system or instrument in concerned with GIS. In part III,we will show how we construct our system ,and how to deploy it in cloud platform. we will show one of our instance in part IV and conclusion in part VI.

\section{Design of lightweight distributed geographic system}

\section{A. Architecture}

A typical architecture of web GIS consist of two big components: client (Browser) and web map server. Though have much in common, architecture of web map server differ from one anther[4].

our web map server undertook by services of our cloud platform using the data both in Hbase[5] and file management clusters. We support plenty of services on our platform, of which one service called "map service" play the role of map server . It is obvious that a service deployed on cluster would be more robust, Scalable, and Stable .

Figure 1 show us the logic architecture of our system briefly. The system can be divided into three level : date level, service level, and user interface level. The three parts of system are relatively independent of each other,they exchange information by invoking standard API. 


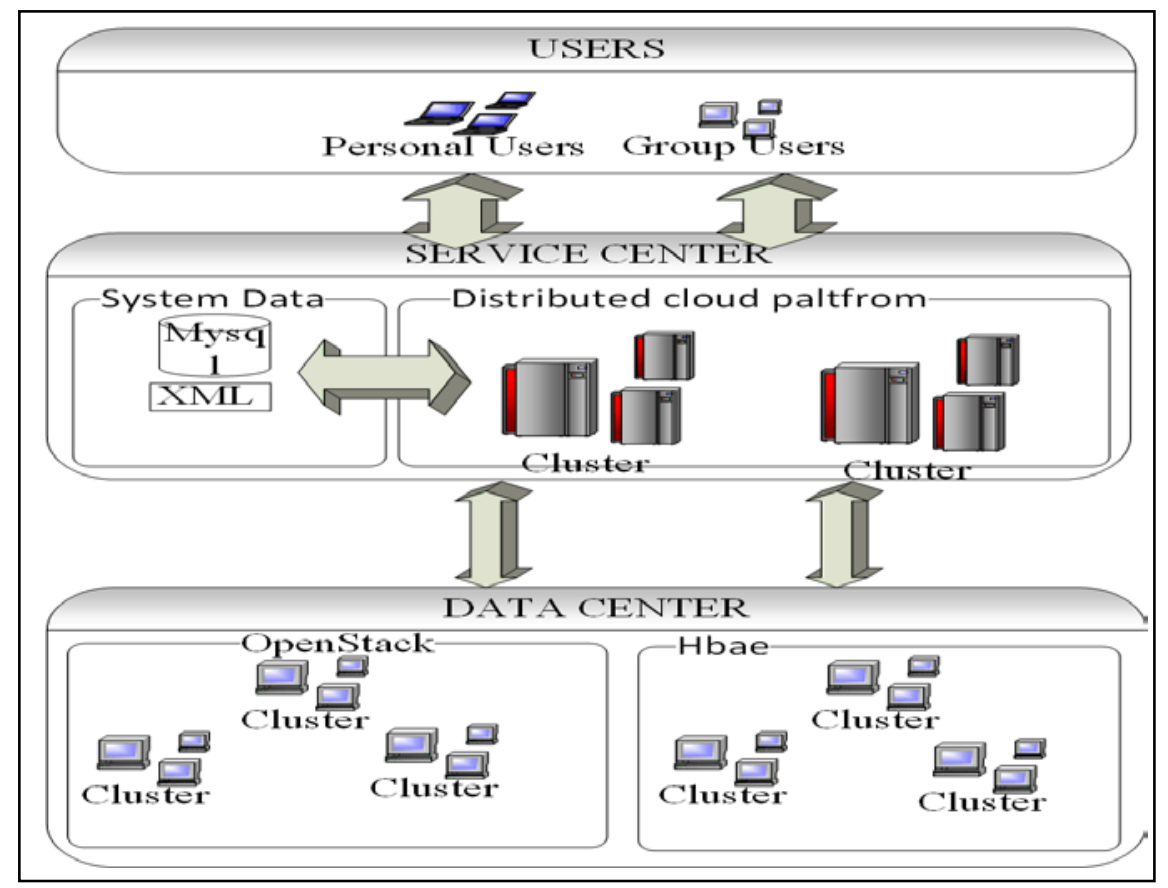

Figure 1. Architecture

\section{1) Data center}

Our data consist of map data,service information ,and mass of user's data marked with geographic meta tags.

Map date are millions of picture in Portable Network Graphic Format. All these map are in size of 256px *256px. Each picture called a tile .There are internal relation in content of the map tiles. Just as the figure 2 elucidated ,ech grid is a tile. Tiles in different level constitute a map of an area in different accuracy level. 


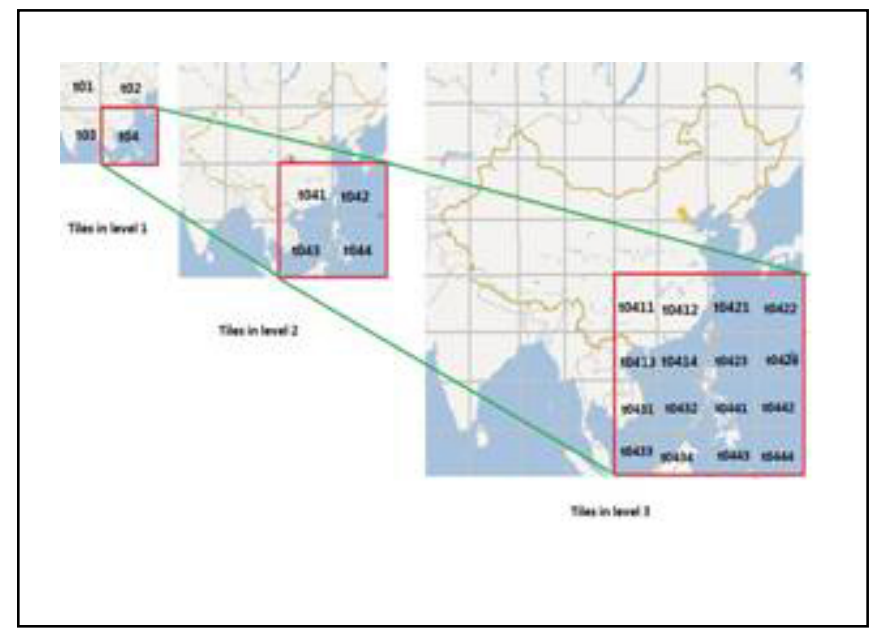

Figure 2. Relation Between Tiles

we select the boundary of map at $2 \mathrm{~N}$ to $54 \mathrm{~N}$, (latitude) $72 \mathrm{E}$ to

136E(longitude), which contains the whole China's territory. We create our tile map in following steps:

(1)We divide the primary picture into pictures in size of 256px*256px as tile map level 1.

(2)Half the plotting scale and then divide the each pictures in last level into four pictures ,they are in size of $256 * 256$,too. These picture constitute the next level.

(3)Repeat the step (2), until we build map to level 12.

It is obvious that the count tile map grow at speed of exponential grows. The number of tiles can be calculated in the follow equation:

In our example ,we set the max-level equals 11 . According to the equation

$$
\mathrm{N}=\sum_{k=0}^{k=n} \boldsymbol{4}^{k}=\frac{4^{n}-1}{3}
$$

(1),the amount of our tile map reaches 100,720.In order to keep high efficiency and security, the marked map files are stored in clusters organized by Openstack[6]. Openstack is good at object store .

User data are variety of geographic meta tags . Our extraction service extract data from difference resource and recognise the geographic meta tags ceaselessly . User data are integrated in Hbase . Distributed non relational database perform well in complexed data integration[7].

2) Web map service 
Map date are millions of picture in Portable Network Graphic Format . Knowing latitude and longitude of the boundary of map area,we can reckon latitude and longitude of each tile map. Given this, we can also know which tiles can joint a map area limited by atitude and longitude.

When showing map ,Each zoom use the tile picture belonged to the right level to ensure the distinguish-ability.

The earth is approximative globe ,there exists distortion when we projection the surface profile to a quadrate paper. Take longitude for example ,they parallel each other in map , but they converge at north pole and south pole as a matter of fact. When we locate a point by latitude and longitude, or get latitude and longitude of an point on our map, we consider the deviation on each level and correct it properly by an empirical value.

Map service receive HTTP request and return the right pictures to client.

\section{3) Data service}

User data are abstract into different data layers.the data layers neither change the logical construction nor storage organization.

Our Map Service receive and analyze the HTTP request. The HTTP request describe the coordinate of meta picture and the boundary of map, by Analyzing the which we compute the serial number of tile picture it needed,and return the certain picture.

Data service also receive and analyze the HTTP request. By analyzing the data layers, we get an position information and several names of data layers. Using these information, we query the HBase to get text, pictures, videos, and other file Associated to the right position and limited by layers in the meantime. We feedback the result in XML file. The Openlayers will display the meta data to decorate the map.

\section{B. Deploy construction}

\section{1) Service cluster}

Service cluster are deployed on virtual machines. high-powered servers are luxurious if used to be clients in cluster. We use vSphere to virtualiza these work station into serval virtual machine to organize an cluster for service provide an dispatch.the whole cluster share a disk array via fiber-optic network.

2) Data and compute cluster

Our data are organized in Hbase cluluser. More than ten machines with hadoop installed compose our data and compute cluster. Different kinds of data concerned with geographic information tags are stored in Hbase. Data cluster manage these data and provide index service and query service . Compute cluster 
receive the request form service cluster , query the Habse , and then sort , filtrate , or cluster , and return the result.

\section{Experiments and experimental Analysis}

To verify our system design, we deployed an instance. Here are our machines:

TABLE I. MACHINE CONFIGURATION

\begin{tabular}{|l|l|r|l|c|}
\hline machine & RAM & CPU & Frequence & Amount \\
\hline PC & 1GB & 1 & $1.8 \mathrm{GHZ}$ & 12 \\
\hline Server & $16 \mathrm{~GB}$ & 4 & $2.8 \mathrm{GHZ}$ & 3 \\
\hline
\end{tabular}

Besides ,we used a fiber switch with 24 port and a gigabit switch with 24 port to construct net work. We install vSphere 5.1 on the servers for virtualization.

TABLE II. OUR CLUSTERS :

\begin{tabular}{|c|c|c|c|}
\hline Cluster & Client & Os & Net work \\
\hline Service & 6 & Ubuntu,windows xp & Fiber \\
\hline Data\&compute & 12 & Ubuntu server & gigabit \\
\hline
\end{tabular}

In our data center, we integrated some biological characteristic data and traditional minority sports event data. Every data layer covers a field. Each item fixed on a point on map .we cluster these points into some group by their position and map zoom. Circles in different size expression the groups and reveal the law of the items's distribution.

In this view, we can see how traditional minority sports event distribute and get their details easily .

\section{Conclusions}

In this article, a lightweight distributed geographic information system solution are presented. We designed the the construction of the system , and put forward an instance to show how it can works and how it could be.This system runs well in our platform.

As a lightweight system, it can be integrated in many other cloud platform easily.

As we described ,this system is co-dependent. We will optimize the performance of data service and enrich the mode of data service continually. 
We hope to packaging the concerned part into different forms of components to make it much more easier to use .

\section{References}

[1] Zhihui Li; Xiuwan Chen; Chiyuan Li, "Integration of distribute GIS and GPS for inter-regional fire emergency management in Yangtze River Delta," Remote Sensing, Environment and Transportation Engineering (RSETE), 2011 International Conference on , vol., no., pp.3228,3231, 24-26 June 2011

[2] Rui-sheng Jia; Yan Jiang; Hong-mei Sun; Xi-juan Wei, "Research on distributed GIS process modeling and integration," IT in Medicine and Education, 2008. ITME 2008. IEEE International Symposium on , vol., no., pp.33,38, 12-14 Dec. 2008

[3] Xiaolin Lu, "An investigation on service-oriented architecture for constructing distributed Web GIS application," Services Computing, 2005 IEEE International Conference on , vol.1, no., pp.191,197 vol.1, 11-15 July 2005

[4] Xu Qian-qian, "A kind of geographic information system application based on flex API of ArcGIS Server," Advanced Computer Theory and Engineering (ICACTE), 2010 3rd International Conference on , vol.3, no., pp.V3-246,V3-249, 20-22 Aug. 2010

[5] Bandopadhyay, S.; Ghosh, A.; Sarkar, R., "Design of an efficient distributed GIS application," TENCON 2003. Conference on Convergent Technologies for the Asia-Pacific Region, vol.3, no., pp.1162,1166 Vol.3, 15-17 Oct. 2003

[6] Vora, M.N., "Hadoop-HBase for large-scale data," Computer Science and Network Technology (ICCSNT), 2011 International Conference on , vol.1, no., pp.601,605, 24-26 Dec. 2011 
[7] Khan, R.H.; Ylitalo, J.; Ahmed, A.S., "OpenID authentication as a service in OpenStack," Information Assurance and Security (IAS), 2011 7th International Conference on , vol., no., pp.372,377, 5-8 Dec. 2011 